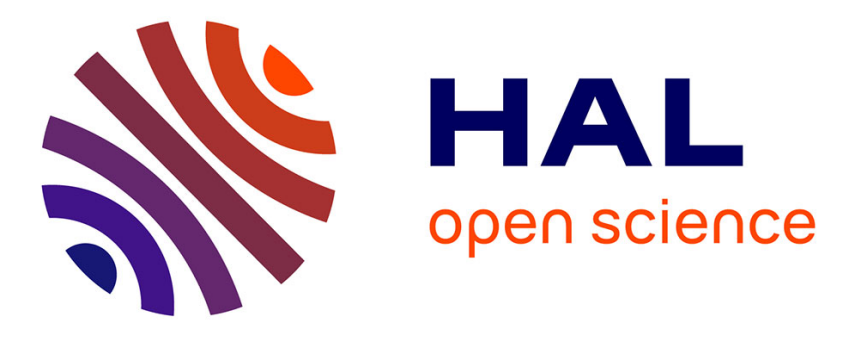

\title{
Topographically induced internal solitary waves in a pycnocline: Ultrasonic probes and stereo-correlation measurements
}

\author{
Yvan Dossmann, Alexandre Paci, Francis Auclair, Mathieu Lepilliez, \\ Emmanuel Cid
}

\section{To cite this version:}

Yvan Dossmann, Alexandre Paci, Francis Auclair, Mathieu Lepilliez, Emmanuel Cid. Topographically induced internal solitary waves in a pycnocline: Ultrasonic probes and stereo-correlation measurements. Physics of Fluids, 2014, vol. 26 (n 5), pp.1-22. 10.1063/1.4873202 . hal-01102330

\section{HAL Id: hal-01102330 \\ https://hal.science/hal-01102330}

Submitted on 13 Jan 2015

HAL is a multi-disciplinary open access archive for the deposit and dissemination of scientific research documents, whether they are published or not. The documents may come from teaching and research institutions in France or abroad, or from public or private research centers.
L'archive ouverte pluridisciplinaire HAL, est destinée au dépôt et à la diffusion de documents scientifiques de niveau recherche, publiés ou non, émanant des établissements d'enseignement et de recherche français ou étrangers, des laboratoires publics ou privés. 


\section{Open Archive TOULOUSE Archive Ouverte (OATAO)}

OATAO is an open access repository that collects the work of Toulouse researchers and makes it freely available over the web where possible.

This is an author-deposited version published in : http://oatao.univ-toulouse.fr/ Eprints ID : 12283

To link to this article : doi:10.1063/1.4873202

URL : http://dx.doi.org/10.1063/1.4873202

To cite this version : Dossmann, Yvan and Paci, Alexandre and Auclair, Francis and Lepilliez, Mathieu and Cid, Emmanuel Topographically induced internal solitary waves in a pycnocline: Ultrasonic probes and stereo-correlation measurements. (2014) Physics of Fluids, vol. 26 ( $\mathrm{n}^{\circ}$ 5). ISSN 1070-6631

Any correspondance concerning this service should be sent to the repository administrator: staff-oatao@ listes-diff.inp-toulouse.fr 


\title{
Topographically induced internal solitary waves in a pycnocline: Ultrasonic probes and stereo-correlation measurements
}

\author{
Yvan Dossmann, ${ }^{1,2,3, a)}$ Alexandre Paci, ${ }^{2}$ Francis Auclair, ${ }^{3}$ \\ Mathieu Lepilliez, ${ }^{2,3,4}$ and Emmanuel $\mathrm{Cid}^{5}$ \\ ${ }^{1}$ Research School of Earth Sciences, The Australian National University, \\ Canberra 0200, Australia \\ ${ }^{2}$ CNRM-GAME, UMR3589 METEO-FRANCE and CNRS, 42 avenue Gaspard Coriolis, \\ 31057 Toulouse Cedex 01, France \\ ${ }^{3}$ Laboratoire d'Aérologie, 14 avenue Edouard Belin, 31400 Toulouse, France \\ ${ }^{4}$ Institut de Mécanique des Fluides de Toulouse, 2 Allée Camille Soula, \\ F-31400 Toulouse, France \\ ${ }^{5}$ Laboratoire de Génie Chimique, UMR5503 INPT, UPS, CNRS, 4, Allée Emile Monso, \\ F-31030 Toulouse, France
}

\begin{abstract}
Internal solitary waves (ISWs) are large amplitude stable waves propagating in regions of high density gradients such as the ocean pycnocline. Their dynamics has often been investigated in two-dimensional approaches, however, their three-dimensional evolution is still poorly known. Experiments have been conducted in the large stratified water tank of CNRM-GAME to study the generation of ISWs in two academic configurations inspired by oceanic regimes. First, ultrasonic probes are used to measure the interfacial displacement in the two configurations. In the primary generation case for which the two layers are of constant density, the generation of ISWs is investigated in two series of experiments with varying amplitude and forcing frequency. In the secondary generation case for which the lower layer is stratified, the generation of ISWs from the impact of an internal wave beam on the pycnocline and their subsequent dynamics is studied. The dynamics of ISWs in these two regimes accords well with analytical approaches and numerical simulations performed in analogous configurations. Then, recent developments of a stereo correlation technique are used to describe the three-dimensional structure of propagating ISWs. In the primary generation configuration, small transverse effects are observed in the course of the ISW propagation. In the secondary generation configuration, larger transverse structures are observed in the interfacial waves dynamics. The interaction between interfacial troughs and internal waves propagating in the lower stratified layer are a possible cause for the generation of these structures. The magnitude of these transverse structures is quantified with a nondimensional parameter in the two configurations. They are twice as large in the secondary generation case as in the primary generation case.
\end{abstract}

\section{INTRODUCTION}

Internal solitary waves (ISWs) are nonlinear internal gravity waves commonly observed in geophysical flows. They propagate in regions of strong density vertical gradients that are present in the atmosphere (Tsai, Kanamori, and Artru, ${ }^{1}$ Rottman and Grimshaw ${ }^{2}$ ) and in the ocean (Halpern, ${ }^{3}$ New and Pingree ${ }^{4}$ ). Their remarkable stability is due to the balance between nonlinear effects and dispersive effects induced by nonhydrostaticity and rotation (Helfrich and Melville ${ }^{5}$ ).

a)yvan.dossmann@anu.edu.au 
In the ocean, these waves are trapped at the bottom of the oceanic mixed layer, namely the pycnocline, where they can propagate horizontally over hundreds of kilometers before decaying (New and da Silva ${ }^{6}$ ). Hence, they play a role in the dynamical and biological properties of the mixed layer (Lai et al., ${ }^{7}$ Kantha and Clayson ${ }^{8}$ ). To describe the role played by ISWs on the mixed layer properties, it is necessary to better understand their generation process and their dynamics.

At least two types of ISWs generation have been observed in the ocean. First, they can be induced by the direct interaction between an oscillatory tidal flow and a steep topography, such as the continental shelf in the Celtic Sea (Halpern, ${ }^{3}$ Pingree and Mardell ${ }^{9}$ ) or Pearl Bank in the Sea of Sulu (Apel et al. ${ }^{10}$ ). This interaction provokes large amplitude vertical motions in the pycnocline above the topography. Due to the nonlinearity/dispersion balance, these vertical motions organize in trains of propagating ISWs. The term "Primary generation" and ISW1 is used in the remainder of the paper to refer to this generation mechanism and this ISW type, respectively.

Using observational measurements in the Bay of Biscay, New and Pingree ${ }^{4,11}$ showed the existence of a different ISWs generation mechanism occurring $150 \mathrm{~km}$ away from the continental shelf. By tracking the path followed by internal waves in the lower layer, they concluded that ISWs are generated when internal wave beams emitted at the shelf impinge on the pycnocline. The same mechanism has been observed near the Sofala shelf in the Mozambique Channel (da Silva, New, and Magalhaes ${ }^{12}$ ) and near the Ortegal Promontory in the Southern Bay of Biscay (Azevedo, da Silva, and $\mathrm{New}^{13}$ ). We adopt the terms "Secondary generation" and ISW2 to refer to this mechanism and this ISW type, respectively. This mechanism is also referred to as "local generation" in the literature.

These two generation mechanisms, and the dynamics of the associated ISWs, have been extensively described in two-dimensional configurations. In particular, the analytical studies of Gerkema, ${ }^{14,15}$ and Maugé and Gerkema ${ }^{16}$ extracted important differences in the dynamics of ISW1s and ISW2s. Using a normal mode approach, they observe that ISW1s propagate in a stable unimodal manner. This stable dynamics was observed in 2D direct numerical simulations of the primary generation process (Dossmann, Auclair, and $\mathrm{Paci}^{17}$ ).

On the contrary, ISW2s are subject to more important energy exchanges with other normal modes while they propagate. These energy transfers lead to a downward leaking of energy from the propagating ISW2 to the stratified lower layer (Gerkema, ${ }^{14}$ Akylas et al. ${ }^{18}$ ). The internal wave beam/pycnocline interaction and the subsequent ISW2 propagation and decay were observed in 2D direct numerical simulations using a forcing by internal wave beams with an analytical shape (Grisouard et al. ${ }^{19}$ ) or generated over a topography (Dossmann, Auclair, and $\mathrm{Paci}^{20}$ ).

Selection criteria relying on the topography shape for the primary generation process and on the internal wave structure in the secondary generation process have been proposed. The reader can refer to Dossmann, Auclair, and Paci ${ }^{17,20}$ for an extensive description of these criteria and complementary references about these two processes.

Laboratory experiments have permitted to study the dynamics of ISWs in real flows. Delisi and Orlanski ${ }^{21}$ performed the first experimental study of the interaction of an internal wave beam with the pycnocline. They observed that large interfacial displacements are induced by the internal wave beam impact in a moderate pycnocline regime. 2D Synthetic Schlieren and PIV measurements have been used to characterize the linear scattering of the impinging internal wave ray on the pycnocline (Mathur and Peacock ${ }^{22}$ ) and the effects of a shear in the pycnocline (Wunsch and Brandt ${ }^{23}$ ). Mercier et $a l .{ }^{24}$ combined the use of 2D PIV measurements and ultrasonic probes aligned in the tank axis to describe the second generation process.

Recent experimental developments have been used to study the three-dimensional (3D) structure of internal waves in quasi-linear regimes using stereoscopic PIV technique (Ghaemsaidi and Peacock ${ }^{25}$ ) and tomographic synthetic Schlieren (Hazewinkel, Maas, and Dalziel ${ }^{26}$ ). Lacaze et $_{\text {al. }}{ }^{27}$ used a stereo-correlation technique to study the three-dimensional structure of interfacial waves generated by an axisymmetric obstacle. In a nonlinear regime, Maxworthy ${ }^{28}$ qualitatively described the structure of ISWs generated over a three-dimensional ridge in a horizontal plane using photographies. Structural variations seemingly caused by the ridge shape were observed in the direction transverse to the flow.

It is necessary to provide tools to quantitatively describe the three-dimensional structure of propagating ISWs. It is a step towards a better localization and quantification of the mixing induced 
in the pycnocline by the ISW propagation and breaking. Moreover, these results can provide insights to assess the need of three-dimensional simulations to model the dynamics of ISWs.

The present article aims at describing the three-dimensional dynamics of ISW1s and ISW2s in laboratory experiments. It relies on measurements of local interfacial displacements and of the three-dimensional structure of the interfacial displacement. This work is in continuity with numerical studies of the primary and secondary generation processes (Dossmann, Auclair, and Paci $^{17,20}$ ). While these studies inspired the choice of physical parameters in the present configurations, regimes of higher nonlinearity are expected in the laboratory experiments. In fact, the experimental ridge shape is 8 times steeper than the numerical one for similar amplitude forcings.

Ultrasonic probes are used to study the ISWs local dynamics in two series of experiments in the primary generation configuration, one with varying amplitude and the other with varying forcing frequency. In the secondary generation configuration, probes are disposed longitudinally to describe the different steps of the ISW dynamics and confront them with previous numerical and experimental studies.

Stereo correlation measurements are used for the first time to capture the dynamics of ISWs in a horizontal field, for the two processes. This experimental configuration allows to quantitatively estimate the magnitude of transverse effects in the dynamics of ISW1s and ISW2s, and to provide hints to understand their origin better.

The paper is organized as follows. Section II introduces the experimental configuration and a validation of the stereo correlation technique. Results issued from local measurements of the interfacial displacements for the primary and secondary generation configurations are respectively presented in Secs. III and IV. The three-dimensional structure of interfacial waves in the two configurations is discussed in Sec. V. Conclusions are drawn in Sec. VI.

\section{EXPERIMENTAL SETUP}

\section{A. Experimental arrangement}

The experiments have been carried out in the large stratified water flume at the geophysical fluid dynamics laboratory of CNRM-GAME (Météo-France and CNRS) in Toulouse (France). This facility has been used as a $22 \mathrm{~m}$ long, $1 \mathrm{~m}$ high, $3 \mathrm{~m}$ wide closed glass tank for the present experiments. The stratification is controlled by the salinity, while the laboratory air temperature is regulated at $20^{\circ}$. Water is supplied in the water tank by two pumps connected to two reservoirs, respectively filled with freshwater and brines. The flow of each pump is controlled by a computer to obtain a given density at a given depth.

Two types of stratification have been used in the experiments. For the primary generation experiments (designed by PG0XX, with $X X$ the experiment number), a two-layer stratification, with constant densities $\left(\rho_{1}, \rho_{1}+\Delta \rho\right)$ and depths $\left(h_{1}, h_{2}\right)$ in the upper and lower layers, respectively, is used in a fluid of total depth $H=40 \mathrm{~cm}$, as shown in Figure 1(a). The density profile is measured with a densitymeter AntonPaar that measures the density at a given depth with a precision of $0.5 \mathrm{~kg} / \mathrm{m}^{3}$. The term pycnocline refers to the thin layer of high vertical density gradient observed in Figure 1(a) that supports the propagation of quasi-interfacial internal waves. The pycnocline center is located at $z=-h_{1}=-10 \mathrm{~cm}$, where $z$ is the vertical coordinate directed upwards, with the origin at the free surface. Its typical thickness varies from $\delta_{p}=2.5 \pm 0.5 \mathrm{~cm}$ for the largest density jumps $(\Delta \rho$ $=90 \mathrm{~kg} / \mathrm{m}^{3}, \mathrm{PG} 038$ to PG045) to $\delta_{p}=3.5 \pm 0.5 \mathrm{~cm}$ for the smallest density jumps ( $\Delta \rho=26 \mathrm{~kg} / \mathrm{m}^{3}$, PG046 to PG050).

For the secondary generation experiments (designed by SG0XX, with $X X$ the experiment number), a two-layer stratification is also used in a fluid of total depth $H=40 \mathrm{~cm}$ or $H=80 \mathrm{~cm}$, but this time the density varies linearly in the lower layer, while the upper layer remains homogeneous, as displayed in Figure 1(b). The density jump in the pycnocline, located at $z=-h_{1}=-4 \mathrm{~cm}$ varies between $\Delta \rho=28 \mathrm{~kg} / \mathrm{m}^{3}$ (SG014 to SG017) and $\Delta \rho=50 \mathrm{~kg} / \mathrm{m}^{3}$ (SG010 to SG013), while the Brunt-Väisälä frequency $N_{0}=\sqrt{-g / \rho_{0} \times d \bar{\rho} / d z}$ varies between $N_{0}=0.93 \mathrm{rad} / \mathrm{s}$ (SG042 to $\mathrm{SG053)}$ and $N_{0}=1.59 \mathrm{rad} / \mathrm{s}$ (SG005 to SG009). 

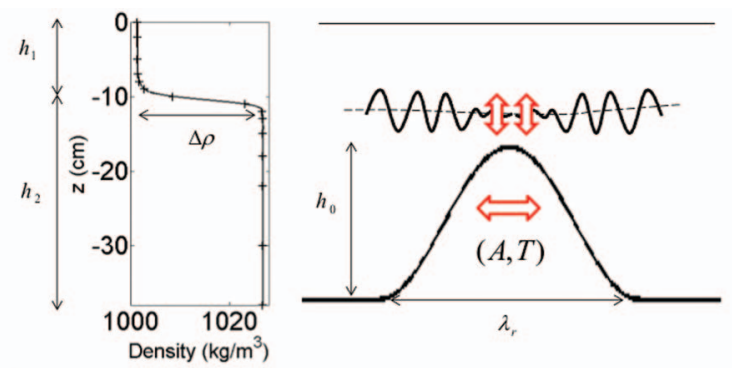

(a)
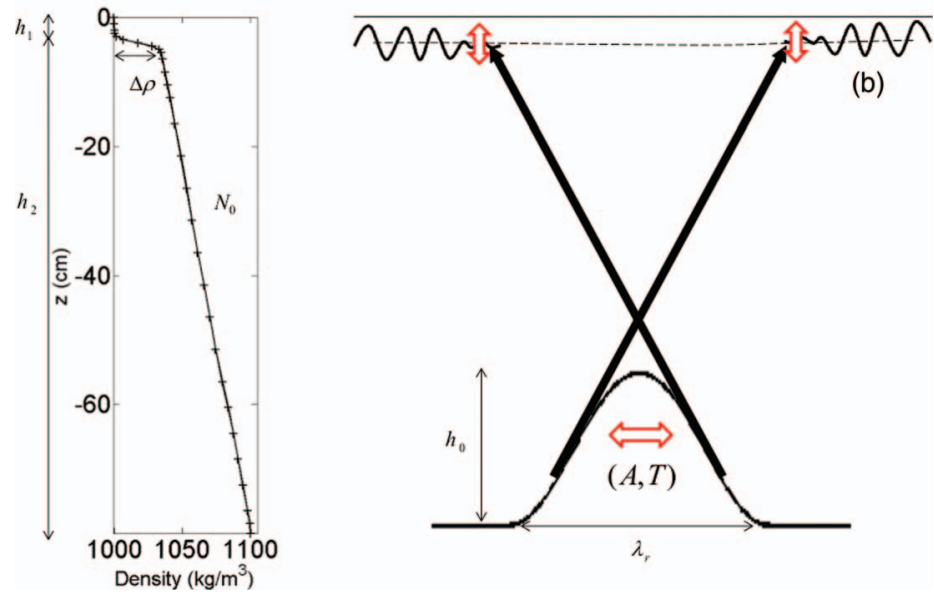

FIG. 1. (a) Configuration for primary generation experiments and the associated characteristic density profile measured by a densitymeter $\left(\Delta \rho=63 \mathrm{~kg} / \mathrm{m}^{3}\right.$, PG027). ISW1s are generated above the ridge crest, located $5 \mathrm{~cm}$ away from the pycnocline rest position. (b) Configuration for secondary generation experiments and the associated characteristic density profile ( $\Delta \rho=33 \mathrm{~kg} / \mathrm{m}^{3}$ in the pycnocline and $N_{0}=0.94 \mathrm{rad} / \mathrm{s}$ in the lower layer, SG023 to SG026). ISW2s are generated by the impingement of topographic internal wave beams on the pycnocline.

In both types of stratification, the water tank is filled from above using eight floating devices. The total flow of $10 \mathrm{~m}^{3} / \mathrm{h}$ used to fill the lower layer is reduced to $3-4 \mathrm{~m}^{3} / \mathrm{h}$ a few centimeters before reaching the pycnocline rest position, in order to limit the vertical extent of the pycnocline. Diffusive effects as well as mixing induced by propagating waves cause a progressive widening of the pycnocline in the course of the experiments. To control the sharpness of the pycnocline, density measurements are performed between series of two to three experiments. The water tank is emptied and refilled when the pycnocline thickness reaches a typical value of $4 \mathrm{~cm}$. For a given stratification five or six experiments can be performed in one to two days.

The forcing is performed by the oscillation of a ridge of sinusoidal shape whose profile is given by

$$
h(x)=1 / 2 h_{0}\left(1+\cos \left(\frac{2 \pi x}{\lambda_{r}}\right)\right),
$$

where $x$ is the horizontal coordinate with the origin at the center of the ridge base, $h_{0}=10 \mathrm{~cm}$ is the ridge height, and $\lambda_{r}=1.25 \mathrm{~m}$ is the ridge bottom width. The ridge spans $2.94 \mathrm{~m}$ in the transverse direction, while the tank width is $2.98 \mathrm{~m}$. The ridge is suspended by four rods, attached on a mobile bench on rails. The mobile bench is linked to a motor forcing a harmonic back and forced motion at precisely controlled frequency and amplitude. Braces are used to prevent supplementary ridge transverse and longitudinal displacements. The ridge displacement is evaluated with a LASER telemeter positioned on one side of the water tank and aiming at a reflector placed on the mobile bench. The Fourier transform of the ridge displacement shows that the forcing is sinusoidal in all experiments, with no visible harmonic peaks.

To study the dynamics of interfacial waves propagating in the pycnocline, local acoustic measurements and three-dimensional optical measurements have been used. 

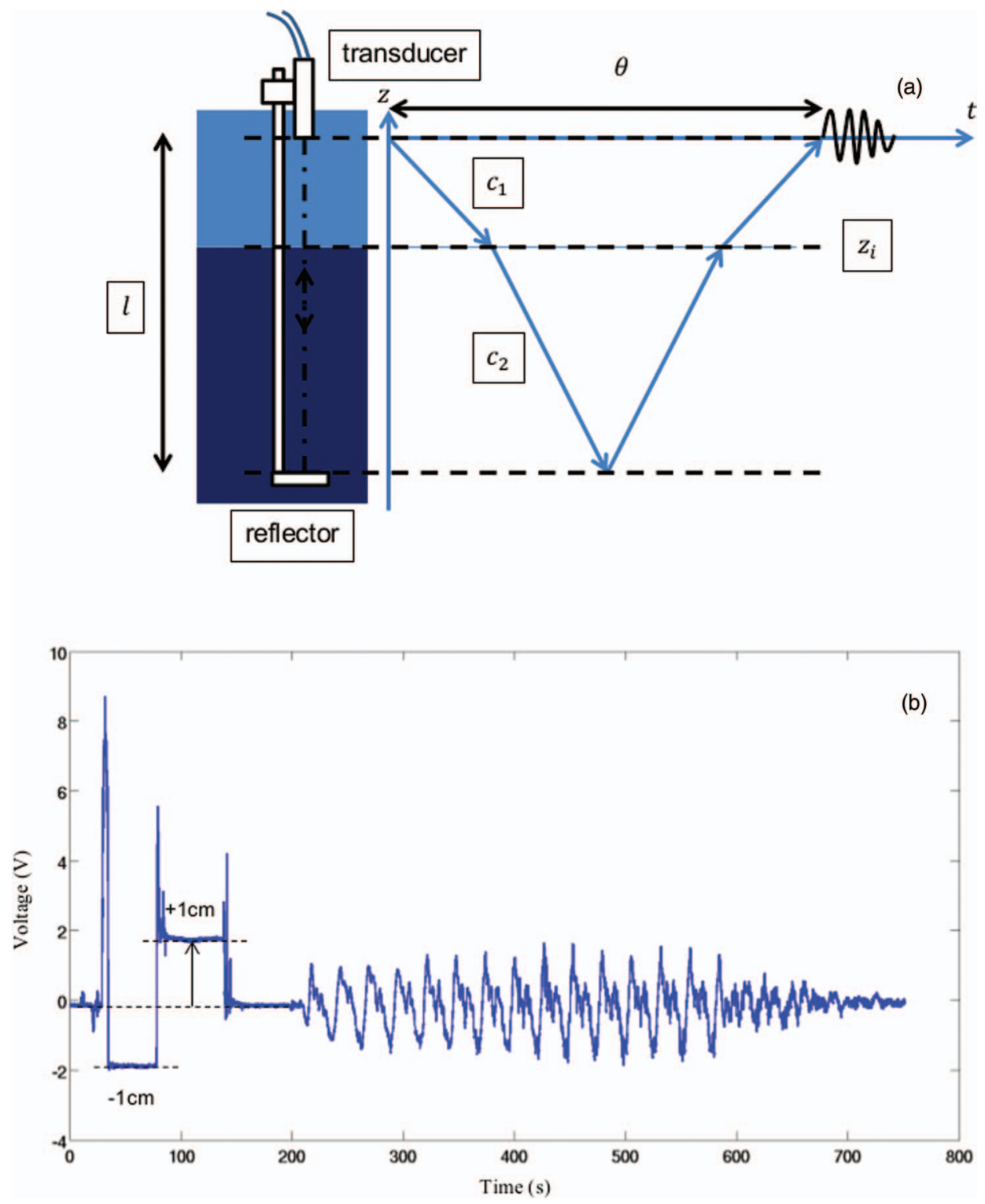

FIG. 2. (a) Scheme of an ultrasonic probe, composed of a transducer (emitter/receiver) located in the upper, lighter layer and a reflector located in the lower, heavier layer. Acoustic pulses are emitted vertically from the transducer to the reflector, where they propagate back to the transducer. $c_{1}$ and $c_{2}>c_{1}$ are the speeds of sound in the upper and lower layers. The timelag $\theta$ varies with the mean vertical displacement between the transducer and the reflector. (b) Raw Voltage output for an experiment with forcing amplitude $A=8 \mathrm{~cm}$ and period $T=25 \mathrm{~s}$. Arrows indicate the displacement/Voltage correspondence issued from the calibration.

\section{B. Ultrasonic probes}

Ten ultrasonic probes have been used to measure the mean pycnocline displacement $z_{i}(t)$ at a frequency of $200 \mathrm{~Hz}$. These probes consist in an emitter-receiver of ultrasonic pulses, and a reflector at a fixed distance $l=15 \mathrm{~cm}$ from the emitter, as shown in Figure 2(a). The device measures the timelag $\theta$ for the pulse to travel on the path emitter-reflector-receiver, which expresses as

$$
\theta=2\left(\frac{l-z_{i}}{c_{1}}+\frac{z_{i}}{c_{2}}\right)
$$

for a pycnocline of zero thickness, where $z_{i}$ is the pycnocline displacement, and $c_{1}$ and $c_{2}>c_{1}$ are the speeds of sound in the upper and lower layers, respectively. The probes deliver a voltage 


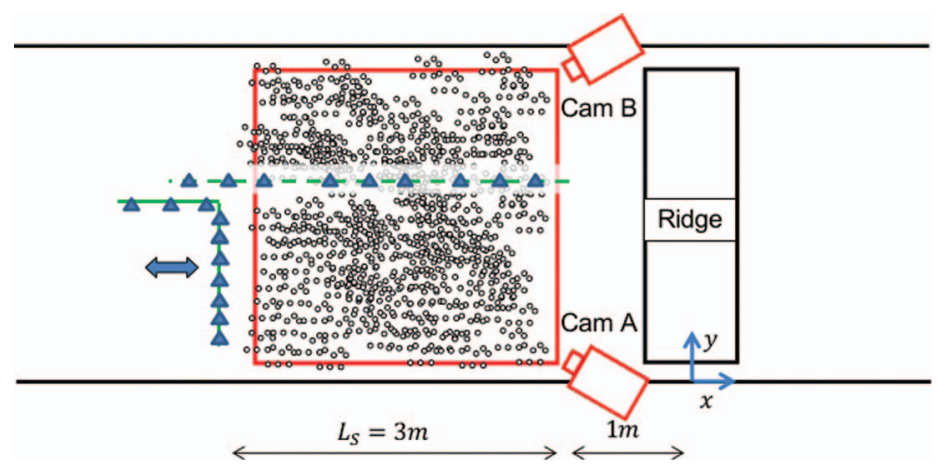

FIG. 3. Experimental arrangement viewed from above. Ultrasonic probes (triangles) are placed on a bench located downstream from the seeded stereo field (square of $3 \mathrm{~m}$ side) during most of the experiments. For seven experiments in the secondary generation configuration, the probes are placed in the tank axis (dashed line).

$V_{s}$ that is proportional to $\theta$, and consequently to the relative distance between the emitter and the pycnocline. In the experimental case, the pycnocline is of finite thickness and the probe measures the mean vertical displacement between the emitter and receiver, which is slightly lower than the maximal vertical displacement in the pycnocline.

Probes are fixed on a bench during the measurements. To calibrate them, the bench is moved $1 \mathrm{~cm}$ up from the measurement position for $1 \mathrm{~min}$, and then $1 \mathrm{~cm}$ down for $1 \mathrm{~min}$ using wedges, at the beginning of a series of experiments. The bench is then placed back to the measurement position before starting the experiment. The Voltage/displacement conversion is then carried out with the calibration measurements, as shown in Figure 2(b). Voltage outputs exhibit weak noise, and the relative uncertainty of the pycnocline displacement estimated at $2 \%$ is mainly due to the calibration step.

During the primary generation experiments, a bench with a "L" shape is used to support the probes as shown in Figure 3. Seven probes are aligned transversally in order to evaluate potential transverse effects in the pycnocline displacement. They are positioned at $x=-3.58 \mathrm{~m}$ and $y=0.12$; $0.34 ; 0.48 ; 0.56 ; 0.75 ; 0.82 ; 0.98 \mathrm{~m}$ where $x$ and $y$ are the longitudinal and transverse coordinates, respectively. $x=0$ (resp. $y=0$ ) is at the center of the ridge average position (resp. at the left edge of the tank in the direction of the wave propagation).

In the secondary generation configuration, eight probes are aligned in the tank axis at $x=-0.23$; $-0.88 ;-1.68 ;-2.08 ;-2.53 ;-2.98 ;-3.43 ;-5.38 \mathrm{~m}$ and $y=1.49 \mathrm{~m}$ as indicated in Figure 3.

\section{Stereo-correlation technique}

This method has been developed to study the evolution of a sediment bed at the bottom of a hydraulic flow by recording and analyzing the structure of a regular pattern projected at the bottom (Astruc et al..$^{29}$ ). It has been adapted to determine the displacement of the pycnocline by observing a random pattern formed by particles seeded in the pycnocline, in experiments performed in the present water tank (Lacaze et $a .^{27}{ }^{27}$ ). The reader may refer to these two articles for a detailed description of this method, for which we recall the main steps here.

The principle of this method is to triangulate the positions of the particles seeded in the pycnocline, observed by two cameras PCO 2000 (4 MPixels) located on both sides of the water tank, and at a height of $4.10 \mathrm{~m}$ with respect to the pycnocline rest position. Cameras are equipped with Nikon Nikkor lenses with a focal length $f=35 \mathrm{~mm}$ and an aperture of 4 . They are inclined with an angle of $20^{\circ}$ with respect to the vertical and the size of the observed field is approximately $3 \mathrm{~m} \times 3 \mathrm{~m}$. Cameras are first calibrated by recording several pictures of a test pattern at the bottom of the empty water tank, in different positions and inclinations. These pictures are used to determine internal parameters of the cameras, such as the focal length and the scale factor, and external parameters such as the position and inclination of one camera with respect to the other.

Before starting an experiment, the pycnocline is seeded with polystyrene particles. A surfactant is added in order to avoid the formation of particles aggregates at the free surface. Pictures are 

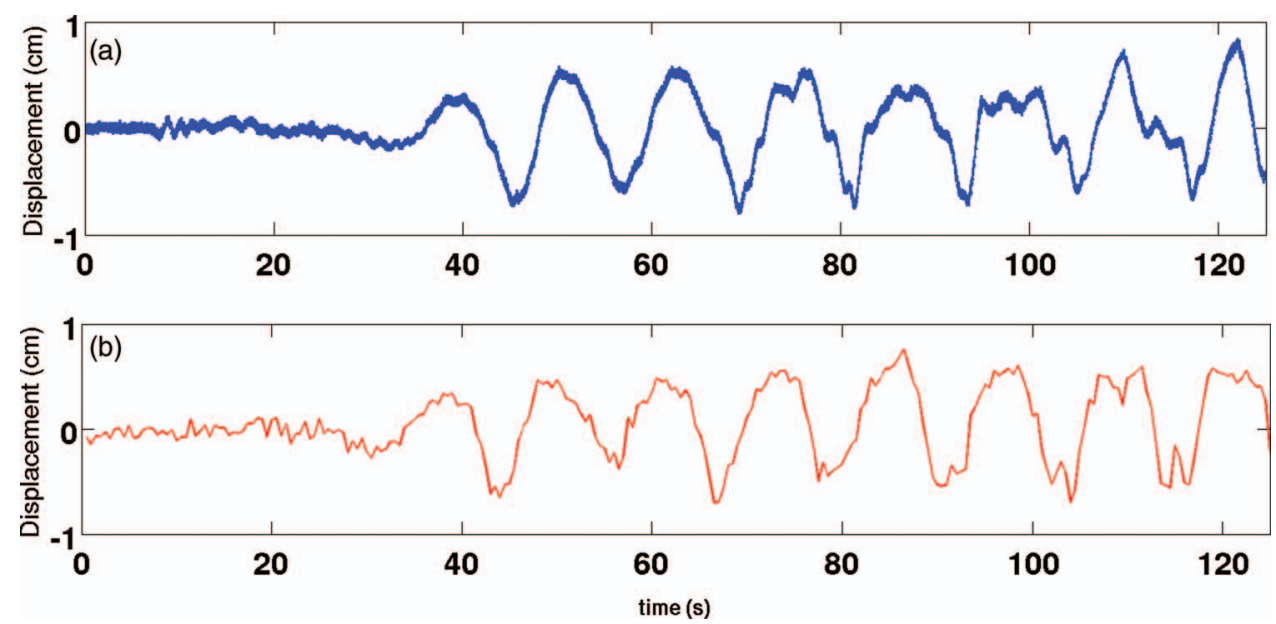

FIG. 4. Simultaneous interfacial displacement measurements with an ultrasonic probe (a) and with the stereo-correlation technique (b). The pycnocline dynamics is similar in the two measurements sets.

synchronously recorded during the experiments, using a low frequency generator sending a square wave at the sampling frequency to the two cameras. The sampling period varies between $200 \mathrm{~ms}$ and $500 \mathrm{~ms}$ so as to obtain 10-20 images pairs per forcing period.

Two steps are then necessary to determine the pycnocline displacements from experimental data. First, images are projected on a common plan to perform an unidimensional particle pairing, which is then used as a forecaster to pair the particles in the raw images. After this pairing step, particles positions are triangulated. Two experiments have been treated with the stereo-correlation technique, one in the primary generation configuration, and the other in the secondary generation configuration.

In the latter case, simultaneous ultrasonic and stereo measurements have been carried out to ensure the consistency of these techniques. For this purpose, the bench supporting the probes has been placed downstream, and just after the stereo field, in experiment SG019. The comparison between time series of the pycnocline displacement measured with one ultrasonic probe on the one hand, and at the closest position of the stereo field to the probe on the other hand, is shown in Figure 4. The two curves are similar, exhibiting periodic upwards and downwards motion with an amplitude of a half-centimeter due to the internal wave propagation in the pycnocline. A short lag of $1.5 \pm 0.1 \mathrm{~s}$ is observed between the two data sets, with the stereo-correlation measurement slightly forward the probe measurement. It corresponds to the theoretical time taken by the wave to propagate over the distance of $20 \mathrm{~cm}$ between the probe and the position of the stereo-correlation measurement, calculated using the wave group speed of $0.15 \mathrm{~m} / \mathrm{s}$.

The correlation coefficient after lag correction and the mean difference between the two measurements are 0.89 and $1 \mathrm{~mm}$, respectively. The stereo correlation technique is therefore sufficiently accurate to capture the dynamics of interfacial displacements on the order of centimetres.

Note that both techniques give access to mode-1 like displacements of the pycnocline. More complex pycnocline displacements are not accessible, however such dynamics has not been observed in video recordings in the present experiments.

In the remainder of the paper, experimental results relying on these two techniques are presented. Experimental parameters of the described experiments and the measurement techniques are recalled in Table I.

\section{PRIMARY GENERATION}

\section{A. Nondimensional parameters}

Nondimensional parameters introduced in previous works (Dossmann, Auclair, and Paci, ${ }^{17}$ Gerkema $^{30}$ ) are used to quantify the strength of nonlinear effects $(\epsilon)$, dispersive effects $(\delta)$, and the 
TABLE I. Experimental parameters.

\begin{tabular}{lcccccccccc}
\hline \hline Series & Experiment & Measurement type & $\Delta \rho\left(\mathrm{kg} / \mathrm{m}^{3}\right)$ & $N_{0}(\mathrm{rad} / \mathrm{s})$ & $A(\mathrm{~cm})$ & $T(\mathrm{~s})$ & $\epsilon$ & $\delta$ & $\lambda_{\text {nd }}$ & $\gamma$ \\
\hline 9 & PG031 & Probe & 60 & $\ll 1$ & 4 & 6 & 0.28 & 3.97 & 1.01 & $\gg 1$ \\
9 & PG032 & Probe & 60 & $\ll 1$ & 6 & 6 & 0.42 & 3.97 & 1.01 & $\gg 1$ \\
9 & PG033 & Probe & 60 & $\ll 1$ & 8 & 6 & 0.57 & 3.97 & 1.01 & $\gg 1$ \\
9 & PG034 & Probe & 60 & $\ll 1$ & 10 & 6 & 0.71 & 3.97 & 1.01 & $\gg 1$ \\
12 & PG040 & Probe & 90 & $\ll 1$ & 8 & 25 & 0.57 & 0.15 & 5.15 & $\gg 1$ \\
12 & PG041 & Probe & 90 & $\ll 1$ & 8 & 18.5 & 0.57 & 0.28 & 3.81 & $\gg 1$ \\
12 & PG042 & Probe & 90 & $\ll 1$ & 8 & 13 & 0.57 & 0.56 & 2.69 & $\gg 1$ \\
12 & PG043 & Probe & 90 & $\ll 1$ & 8 & 15 & 0.57 & 0.42 & 3.09 & $\gg 1$ \\
12 & PG044 & Probe & 90 & $\ll 1$ & 8 & 12 & 0.57 & 0.66 & 2.47 & $\gg 1$ \\
12 & PG045 & Probe & 90 & $\ll 1$ & 8 & 8 & 0.57 & 1.49 & 1.65 & $\gg 1$ \\
18 & PG052 & Stereo & 90 & $\ll 1$ & 8 & 8 & 0.57 & 1.49 & 1.65 & $\gg 1$ \\
19 & SG019 & Probe/Stereo & 30 & 1.23 & 4 & 12 & 0.28 & N/A & N/A & 0.21 \\
21 & SG033 & Probes & 33 & 0.93 & 8 & 13 & 0.28 & N/A & N/A & 0.15 \\
\hline \hline
\end{tabular}

nondimensional wavelength $\lambda_{\text {nd }}$

$$
\begin{gathered}
\epsilon=4.5 \frac{A}{\lambda_{r}} \frac{h_{0}}{H}, \\
\delta=\left(\frac{H \omega}{c^{*}}\right)^{2},
\end{gathered}
$$

and

$$
\lambda_{n d}=\frac{c^{*} \times T}{\lambda_{r}}=\frac{\lambda}{\lambda_{r}}
$$

with $c^{*}=\sqrt{g \frac{\Delta \rho}{\rho_{0}} \frac{h_{1} h_{2}}{H}}$ the linear longwave speed for interfacial waves in the case of a zero thickness pycnocline, $\omega=2 \pi / T$ the forcing pulsation, and $\lambda=c^{*} \times T$ the theoretical linear longwave speed. In a given series of experiments, the typical nondimensional distance between two successive interfacial waves $\lambda_{n d}$ is varied via the forcing period $T$. Larger (smaller) wavelengths correspond to weaker (stronger) dispersive effects.

The length scale $\lambda_{r} / 4.5$ is used in the nonlinearity parameter $\epsilon$. It is the e-folding width of a Gaussian ridge with the same maximum slope as the experimental ridge described by Eq. (1). Using this length scale, the parameter $\epsilon$ can be directly compared to the one used in simulations with a Gaussian ridge (Dossmann, Auclair, and $\mathrm{Paci}^{17}$ ).

\section{B. Strong dispersion}

In series 9, the effect of an increasing nonlinearity parameter for a constant dispersion parameter is examined. Only weak transverse variations in the interfacial displacements are observed with the different probes, therefore the focus is put on the interfacial displacement measured by the probe located at $x=-3.58 \mathrm{~m}$ and $y=0.48 \mathrm{~m}$. In Figure 5 is shown the interfacial displacement for the four experiments of this series along with their corresponding Fourier Transform.

In all experiments of the series, the interfacial wave increases in amplitude in the two first forcing periods. The wave structure is then conserved over 15 periods. After that time, interactions between the emitted and reflected waves affect significantly the wave structure. The time window for the Fourier Transforms starts four periods after the interfacial wave reaches the probe and includes 10 periods in the steady regime.

The interfacial displacement has a similar evolution in the four experiments. About $2 \mathrm{~s}$ after starting the ridge oscillation, a periodic interfacial displacement on the order of millimetres reaches the probe. It corresponds with the propagation of surface waves at a speed $\sqrt{g H} \approx 2 \mathrm{~m} / \mathrm{s}$. 

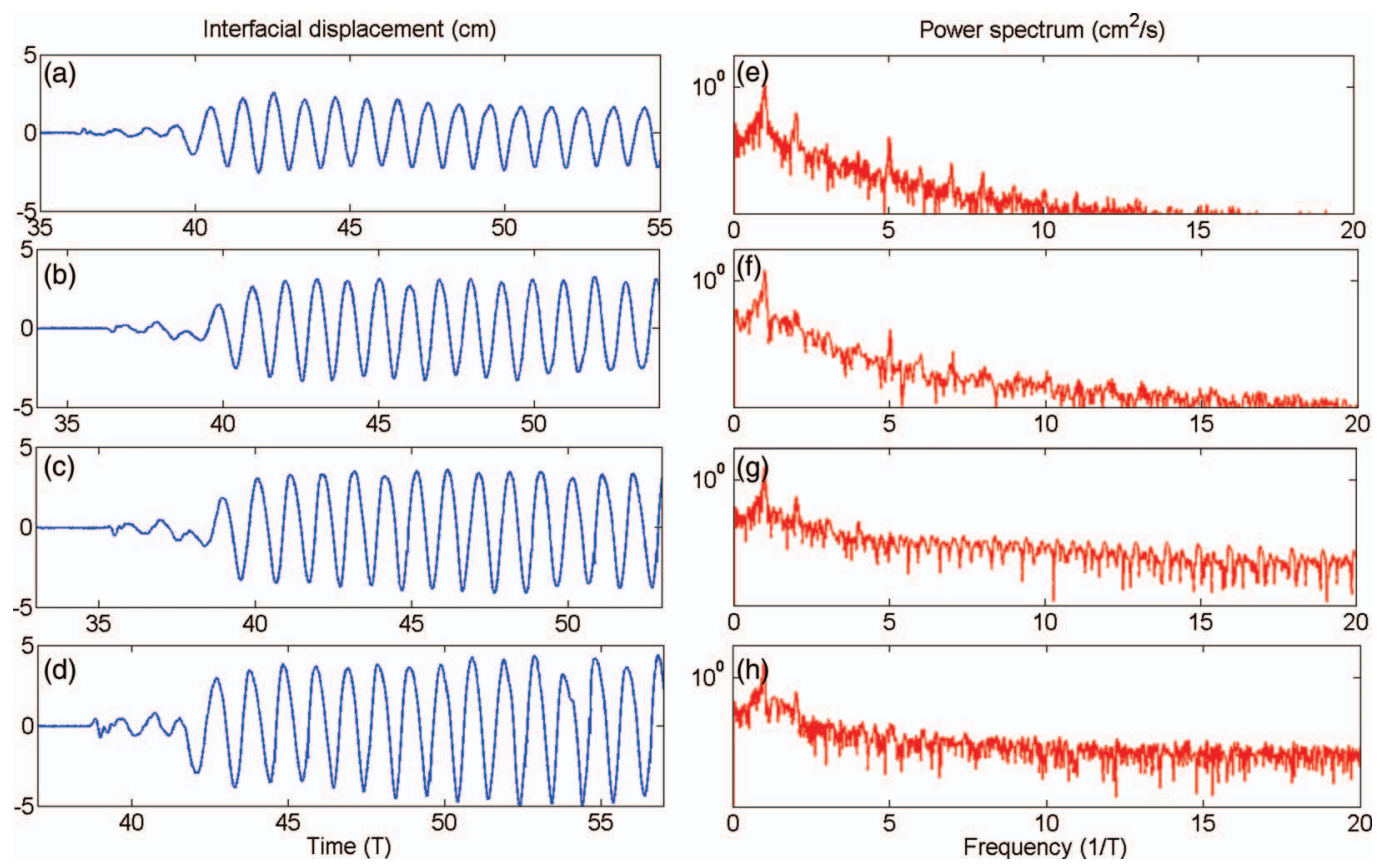

FIG. 5. Pycnocline displacement and corresponding Fourier spectrum in a series of experiments PG031 (a) and (e), PG032 (b) and (f), PG033 (c) and (g), and PG034 (d) and (h) with $\lambda_{n d}=1.01$ and increasing amplitude from top to bottom. The time origin corresponds to the beginning of the calibration.

Three periods later, periodic pycnocline displacements on the order of centimetres are observed. This delay of three periods corresponds with the theoretical time of $18 \mathrm{~s}$ needed for the pycnocline wave to reach the probe, calculated from $c^{*}$ and the ridge-probe distance. The wave amplitude increases with the forcing amplitude from $2.0 \pm 0.1 \mathrm{~cm}$ (PG031, $\epsilon=0.28$ ) to $4.7 \pm 0.1 \mathrm{~cm}$ (PG034, $\epsilon=0.71)$. The pycnocline waves are weakly asymmetrical, as the waves fronts are slightly steeper than the back of the waves. The Fourier spectra indicate that the pycnocline wave is overall sinusoidal, as the first harmonics contributes to less than $5 \%$ of the total signal, and higher order harmonics are hardly visible. In the two last experiments, interfacial waves are less regular in shape than for experiments with a smaller amplitude. This can be caused by wave breaking observed during the experiments.

As the waves amplitude are at the same order of magnitude as the pycnocline depth, nonlinear effects are at play in these experiments. The nonlinearity and dispersion parameters are at least one order of magnitude greater than the ones obtained in the study of Dossmann, Auclair, and Paci $^{17}$ for which trains of at least two ISW1s were observed (e.g., their Figure 3). In series 9, one stable interfacial wave is formed per forcing period. We infer that the strong nonlinearity of the waves is balanced by strong dispersive effects, preventing them to break into ISW1s trains. Regimes with weaker dispersion, for which ISW1s trains are likelier to be observed, are studied in Subsection III C.

\section{Weak dispersion}

In order to investigate the potential breaking of interfacial waves into trains of ISW1s, the study of the series 12 is carried out. In Figure 6 is shown the interfacial displacements for the six experiments in the series 12. The dispersion parameter decreases from Fig. 6(a) (PG045, $\delta=1.49$ ) to Fig. 6(f) (PG040, $\delta=0.15$ ), while the nonlinearity parameter is constant $(\epsilon=0.57)$.

In experiment PG045, a periodic asymmetric wave showing flat tops and narrow troughs is observed after a transient regime lasting $1 \mathrm{~T}$. The troughs have a depth of $6.4 \pm 0.1 \mathrm{~cm}$, which is of the same order of magnitude of the upper layer depth $\left(h_{1}=10 \mathrm{~cm}\right)$. When decreasing the 

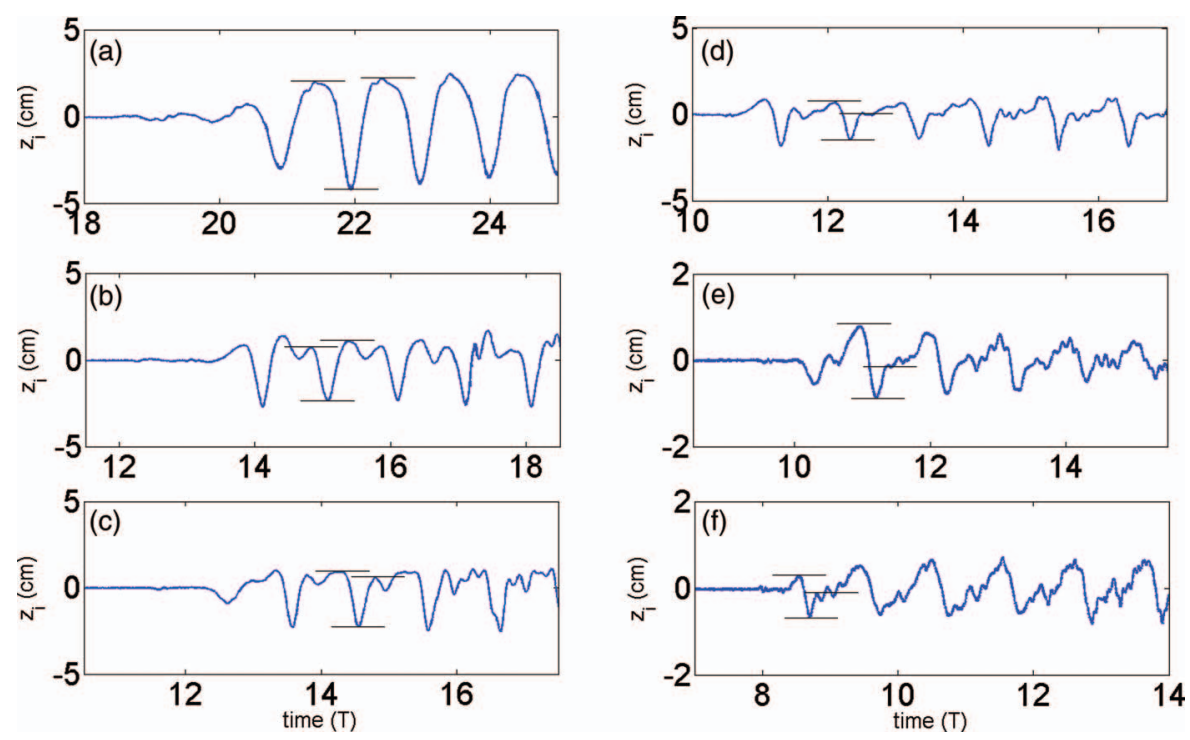

FIG. 6. Pycnocline displacement in a series of experiments PG045 (a), PG044 (b), PG042 (c), PG043 (d), PG041 (e), and PG040 (f) with $\epsilon=0.57$ and decreasing $\delta$ (increasing $\lambda_{n d}$ ) from (a) to (f).

dispersion parameter, the interfacial wave splits into periodic trains of two or three troughs ranked by decreasing depth as seen in PG044 $(\delta=0.66)$ and PG042 $(\delta=0.56)$. For these two experiments, the depth of the first trough is twice as small as in experiment PG045, while the second trough is typically $5 \mathrm{~mm}$ deep. Note that the number of troughs can vary after three forcing periods due to transient effects and interactions with reflected waves. The number of troughs in a train increases with decreasing dispersion parameter: three to four troughs are observed in PG043 $(\delta=0.42)$ and PG041 $(\delta=0.28)$ and four troughs are observed in PG040 $(\delta=0.15)$. For these three experiments, the first troughs are typically $1 \mathrm{~cm}$ deep, and the following troughs in the train have depths on the order of millimetres.

Figure 7 shows the evolution of the depth of the first trough in a train with respect to $\delta$. The wave symmetry is evaluated by measuring the trough depth with respect to the front and to the back of the trough. The trough depth increases monotonically with the dispersion parameter and it becomes more symmetrical.

\section{Nature of the interfacial waves}

In series 9 and 12, the amplitude of the wave or the depth of the first trough in a train are comparable to the pycnocline depth. Therefore, substantial nonlinear effects are at play in the propagation of interfacial waves. These effects are associated with a steepening of the interfacial wave that splits up into a trains of depth-ordered troughs in the experiments with relatively small dispersion parameter (PG040, PG041, PG042, PG043, PG044). Probe measurements carried out $1 \mathrm{~m}$ away in the direction of propagation and the stereo-correlation measurements presented in Sec. V showed that the train structure is overall conserved during the propagation. The balance between nonlinear and dispersive effects can explain this stability. In a qualitative way, these interfacial troughs propagate as ISW1s.

In experiments with larger dispersion parameter (PG045, PG031, PG032, PG033, PG034), we infer that dispersive effects are sufficiently strong to sustain the propagation of a periodic nonlinear interfacial wave. Its structure, consisting in a flatter top and a narrower trough, is similar to the one of the cnoidal waves described in Gerkema, ${ }^{30}$ Chap. 1.2.3.

For series 12, we compare the number of observed troughs per train with the expected number of ISW1s $N_{t h}$, expressed in terms of $\epsilon$ and $\delta$ in Eq. (6), obtained by Gerkema ${ }^{30}$ in the case of 


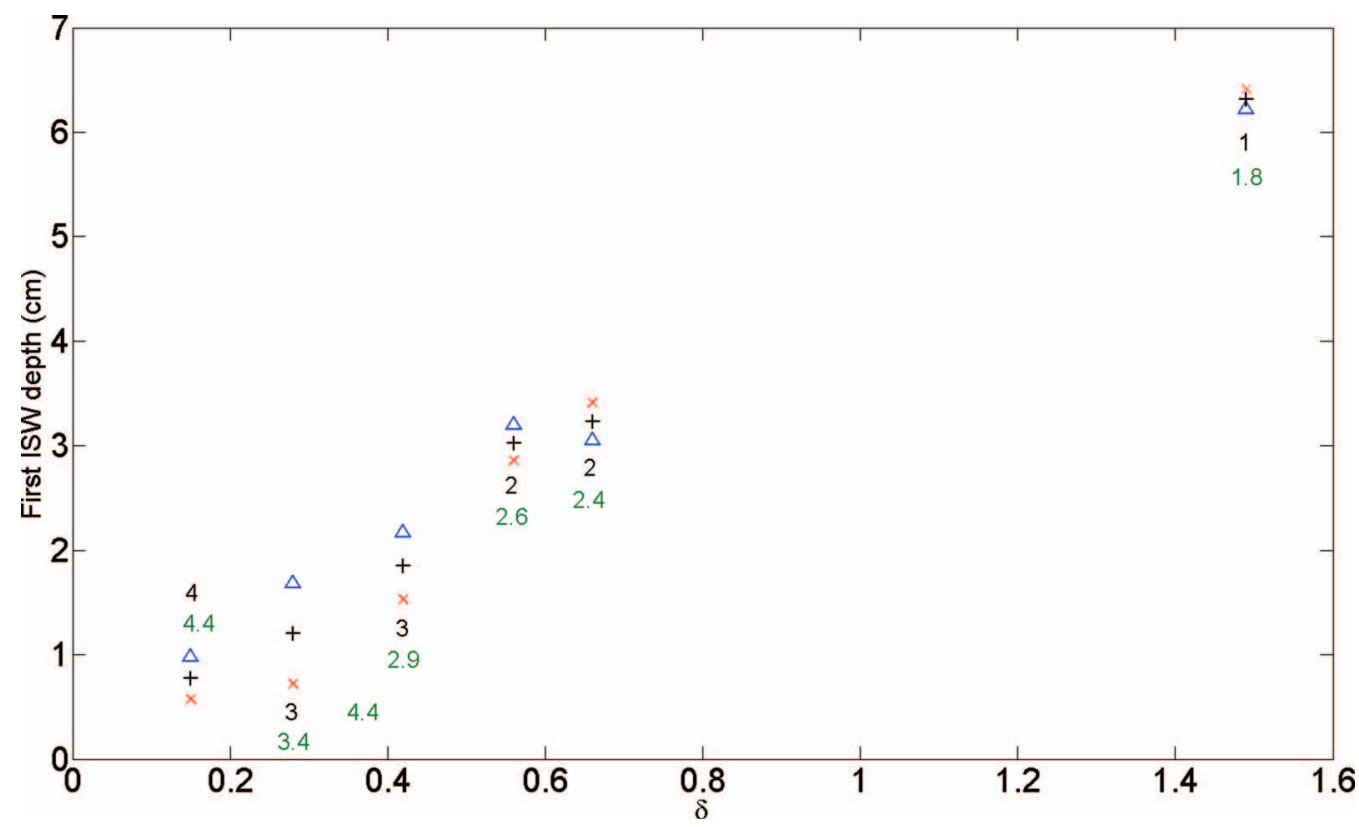

FIG. 7. Evolution of the first ISW1 depth in a train with respect to the dispersion parameter $\delta$. Triangles (resp. crosses) show the depth measurements with respect to the front (resp. to the back) of the wave. + show the depth average values. For each experiment, the first number is the number of ISW1s observed in a train. The second number is the expected number of ISW1s per train using Eq. (6).

an infinitely thin pycnocline

$$
N_{t h}=\frac{1}{2}\left(\sqrt{1+\frac{6 a \epsilon(1-2 \alpha)}{\alpha(1-\alpha) \delta}}+1\right) \text {, }
$$

with $\alpha=h_{1} / H$ and $a=0.5$, the typical ISW1 depth of $5 \mathrm{~cm}$ nondimensionalized by $h_{1}$. Green numbers in Figure 7 stand for $N_{t h}$, whose round value corresponds with the theoretical ISW1s number in a train. The actual number of troughs in a train is in good accordance with $N_{t h}$, although it is slightly overestimated in the theoretical approach. Viscous effects and the pycnocline finite thickness, that are not accounted for in Gerkema's ${ }^{30}$ theory, could explain this discrepancy.

In the numerical study of Dossmann, Auclair, and Paci, ${ }^{17}$ with an identical configuration except for the ridge steepness, similar trains are observed with a nonlinearity parameter $\epsilon$ one order of magnitude lower (e.g., Figure 6(b) and their Figure 3). These interfacial waves were identified as ISW1s by comparing their shape with outputs of a numerical solution of the Korteweg-de Vries equation.

The overall observed dynamics and the strong similarities with one analytical approach and the associated numerical study lead us to associate the propagating troughs with ISW1s in the present experiments. We choose to use this term to describe cnoidal waves as well, since their dynamics is also led by the nonlinearity/dispersion balance.

The typical ISW1s depths and nonlinearity parameters are $5 \mathrm{~cm}$ and $\epsilon=0.5$ in the present experiments and $2 \mathrm{~cm}$ and $\epsilon=0.05$ in the numerical simulations of Dossmann, Auclair, and Paci. ${ }^{17}$ Therefore, the primary generation process appears to be more efficient in the latter case. The mixing observed in the pycnocline above the ridge during the experiments could explain this efficiency decrease in highly nonlinear regimes. In fact, one substantial fraction of the forcing energy is directly transferred to the turbulent scales near the ridge, and is consequently not transferred to the propagating ISW1s. 


\section{SECONDARY GENERATION}

\section{A. Pycnocline strength}

In the secondary generation configuration, the stratified lower layer can support the propagation of topographically generated internal wave beams. When the beam impinges on the pycnocline, it can induce large vertical displacements that can evolve into ISW2s. The efficiency of the secondary generation process is controlled by the "strength" of the density jump in the pycnocline with respect to the lower layer stratification. This quantity has been quantified using different nondimensional parameters in previous analytical approaches (Gerkema, ${ }^{14}$ Akylas et al. ${ }^{18}$, Delisi and Orlanski ${ }^{21}$ ). The reader can refer to Dossmann, Auclair, and $\mathrm{Paci}^{20}$ for a description of these parameters and their relation to the efficiency of the secondary generation process.

In the present article, we adopt the nondimensional parameter $\gamma=c^{*} /\left(N_{0} H\right)$ introduced by Gerkema ${ }^{14}$ to describe the pycnocline strength, where $c^{*}$ is the linear interfacial longwave speed in the pycnocline and $N_{0}$ is the Brunt-Väisälä frequency in the lower layer. The generation of large amplitudes ISW2s is observed in regimes of moderate pycnoclines correspond to $\gamma \approx 0.1$ (Grisouard and Staquet, ${ }^{31}$ Mercier et al.,${ }^{24}$ Dossmann, Auclair, and $\mathrm{Paci}^{20}$ ) as predicted by analytical studies (Gerkema, ${ }^{14}$ Akylas et al. ${ }^{18}$ ). In the seven series of experiments carried out in the secondary generation configuration, the stratification profiles correspond to moderate $(\gamma=0.15)$ to relatively strong $(\gamma=0.31)$ pycnocline strengths. The secondary generation process has been observed in this range of pycnocline strengths (Dossmann, Auclair, and $\mathrm{Paci}^{20}$ ). For weaker density jumps, the pycnocline thickness is similar to the upper layer thickness, and cannot be considered as an quasi-interface separated the upper and lower layers. One selected experiment is presented in this section.

\section{B. Longitudinal structure}

Several steps are involved in the secondary generation process: the generation of large vertical displacements at the pycnocline by the beam impact, the subsequent propagation of ISW2s in the pycnocline and a progressive downward leakage of energy. To potentially observe these different steps, eight probes aligned in the direction of the forcing are used in the experiment SG033.

The total depth is twice as large as the depth in the primary generation configurations. It permits to reach a moderate pycnocline strength $\gamma=0.15$ with a sufficient density jump. Moreover, as the ridge is located further away from the pycnocline, vertical displacements directly induced by the ridge oscillation are expected to be weaker than in the primary generation configuration. One can focus better on the secondary generation process.

Figure 8 displays the interfacial displacement and the corresponding Fourier Transform at each position. At $x_{1}$, the ridge oscillation forces a quasi-harmonic interfacial displacement of millimetric amplitude. The pycnocline dynamics is strikingly different at $x_{2}$, where periodic trains of steep troughs are measured. The number of troughs varies between 1 and 4 during the experiment. The depth of the first trough is $6 \mathrm{~cm}$, and the following troughs, when present, are about $5 \mathrm{~mm}$ deep. In the Fourier spectrum, a strong signal at the forcing frequency is measured, and the four following harmonics have a significant amplitude. When propagating away from the ridge, the first trough progressively weakens from a depth of $4.7 \mathrm{~cm}$ at $x_{2}$ to $2 \mathrm{~cm}$ at $x_{7}$, while the wave structure is overall conserved. The trough structure is hardly distinguishable at $x_{8}$.

In order to assess whether the interfacial troughs can be associated with ISW2s, characteristics of the internal wave field in the lower layer are qualitatively estimated in a linear regime. As the Brunt-Väisälä pulsation is constant in the lower layer, linear internal waves propagate as upgoing and downgoing beams. The theoretical angle of the internal wave beams with respect to the horizontal $\theta_{r}$ can be estimated using the dispersion relation $\omega=N_{0} \sin \theta_{r}$, where $\omega$ is the forcing pulsation. One finds $\theta_{r}=31$ for experiment SG033, an angle close to the maximal ridge angle $\theta_{m}=32$. This angle matching corresponds to the near-critical regime of internal wave beams generation, for which large amplitude upgoing, and potentially downgoing, internal wave beams are emitted on either sides of the ridge (Dossmann et al. ${ }^{32}$ ). 

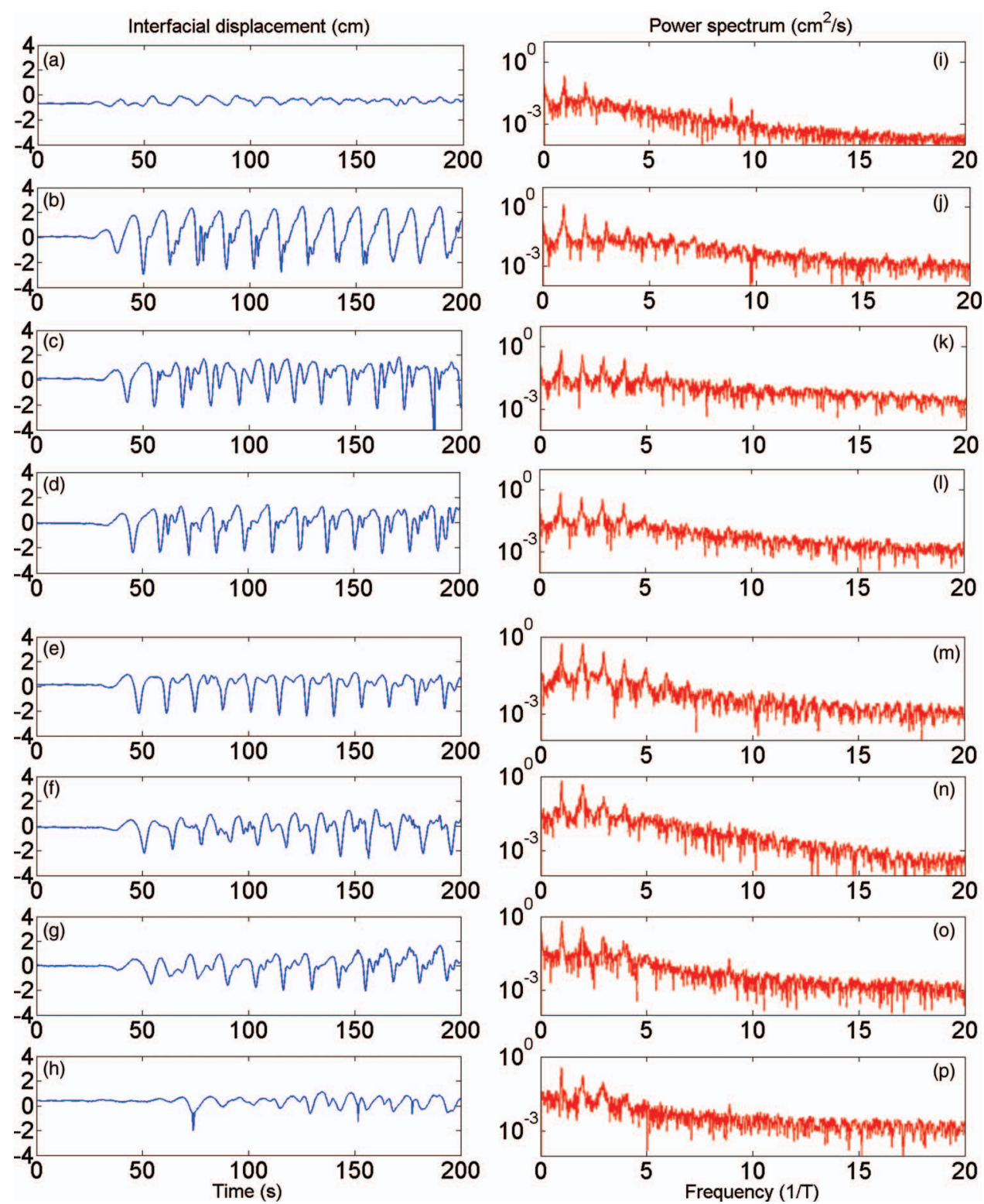

FIG. 8. Pycnocline displacement and corresponding Fourier spectra obtained from eight ultrasonic probes positioned at $x_{1}$ $=-0.23 \mathrm{~m}(\mathrm{a})$ and (i); $x_{2}=-0.88 \mathrm{~m}(\mathrm{~b})$ and (j); $x_{4}=-1.68 \mathrm{~m}(\mathrm{c})$ and $(\mathrm{k}) ; x_{5}=-2.08 \mathrm{~m}(\mathrm{~d})$ and (l); $x_{6}=-2.53 \mathrm{~m}(\mathrm{e})$ and $(\mathrm{m}) ; x_{7}=-2.98 \mathrm{~m}(\mathrm{f})$ and $(\mathrm{n}) ; x_{8}=-3.43 \mathrm{~m}(\mathrm{~g})$ and $(\mathrm{o}) ; x_{9}=-5.38 \mathrm{~m}(\mathrm{~h})$ and $(\mathrm{p})$, where $x=0$ is the ridge rest position. The internal wave beam impacts the pycnocline at $x=x_{i, 1}=-0.75 \pm 0.05 \mathrm{~m}$.

The initially upgoing internal wave beam propagating to the left of the ridge impinges on the pycnocline at $x_{i, 1}=-0.75 \pm 0.05 \mathrm{~m}$. This position is found between $x_{1}$, where the pycnocline is almost non-moving, and $x_{2}$, where nonlinear propagating troughs are observed. The generation of these troughs is likely due to the internal wave beam impact on the pycnocline. They are ranked by decreasing depth and directed towards the thicker layer, which is characteristic of propagating ISWs (e.g., Dauxois and Peyrard ${ }^{33}$ ).

The secondary generation process is apparently at play in the experiment SG033. In this moderate pycnocline regime $(\gamma=0.15)$, a strong linear scattering of the internal wave beam is expected when it impinges on the pycnocline (Gerkema, ${ }^{14}$ Dossmann, Auclair, and Paci, ${ }^{20}$ Mercier et al. ${ }^{24}$ Grisouard and Staquet ${ }^{31}$ ). This process can provoke the generation of large vertical displacements in the pycnocline that organize into ISW2s trains. In a similar (numerical) configuration, ISW2s 


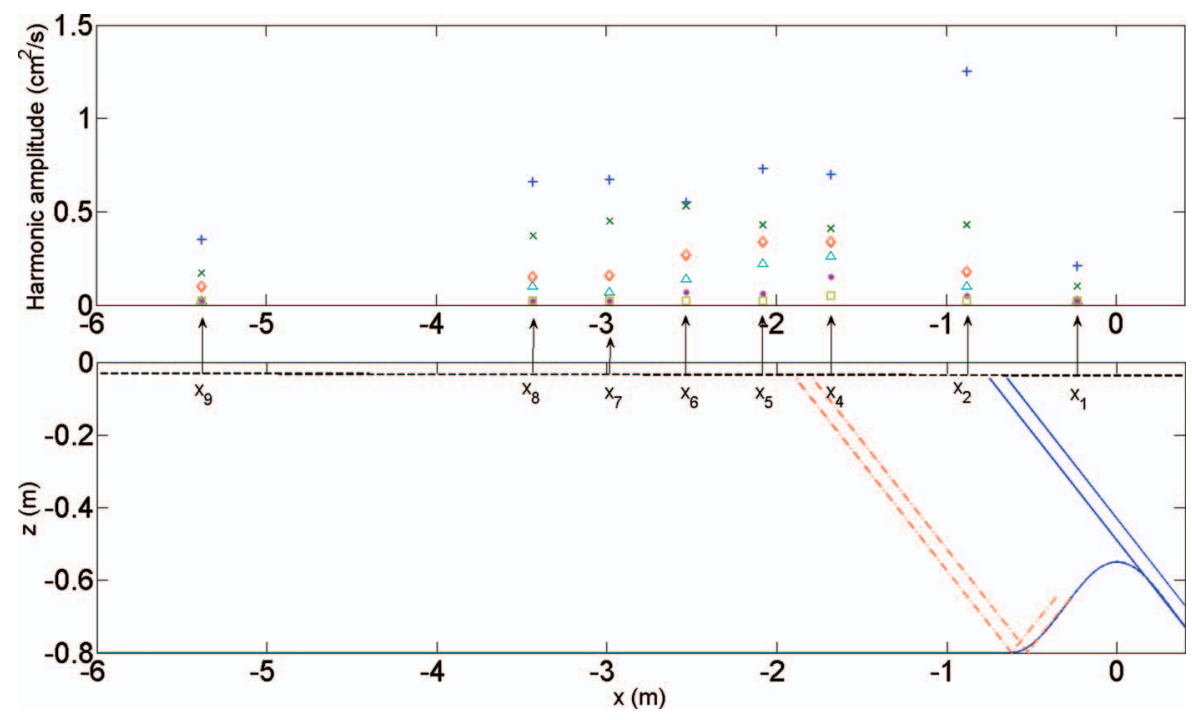

FIG. 9. (a) Evolution of the fundamental ( + signs), first ( $\times$ signs), second (diamonds), third (triangles), fourth (dots), and fifth (squares) harmonics amplitude with respect to the ridge/probe distance. (b) Schematic paths of the initially upgoing (solid lines) and the hypothetical initially downgoing (dashed lines) internal wave beams emitted over the ridge. Arrows indicate the probes positions.

were generated by the impact of a topographic internal wave beam on the pycnocline (Dossmann, Auclair, and Paci ${ }^{20}$ their Figure 3). The ISW2s amplitude was progressively decaying owing to a downward leaking of energy $\left(\right.$ Gerkema $\left.^{14}\right)$, over a typical length scale of $1 \mathrm{~m}$. An amplitude decay is also observed in the present experiment, over a similar scale of $2 \mathrm{~m}$.

Overall, the dynamical properties of the propagating troughs permit to qualitatively identify them as ISW2s.

The evolution of the fundamental and the five first harmonics amplitudes with respect to the measurement position is plotted in Figure 9(a). The amplitudes are relatively small at $x_{1}$, where the internal wave beam has not impacted the pycnocline. The largest value for the fundamental amplitude is reached just after the first impact of the internal wave beam on the pycnocline. After that, it progressively decreases when moving away from the first impact due to the amplitude decay.

If an initially downgoing internal wave beam is emitted, it is supposed to impact on the pycnocline at $x_{i, 2}=-1.85 \pm 0.05 \mathrm{~m}$ after reflecting at the bottom. However, no substantial evolution of the interfacial displacement is observed between $x_{4}=-1.68 \mathrm{~m}$ and $x_{5}=-2.08 \mathrm{~m}$, apart from the amplitude decay. This dynamics suggests that the initially downgoing beam is either absent, or too weak to generate interfacial displacements on the order of centimetres. Similarly, there is no noticeable influence of the subsequent impacts of the initially upgoing internal wave beam on the interfacial displacement. It can be explained by the strong scattering of the beam at the first impact. The beam energy is spread over a larger distance and is therefore not as efficient to generate large interfacial displacements.

ISW2s observed at $x_{4}=-1.68 \mathrm{~m}$ are similar in shape to the one observed by Mercier et al. ${ }^{24}$ in moderate pycnocline regimes (their Figures 11(a) and 11(b)), apart from the ISW2 depth which is one order of magnitude larger in the present experiment. In their experiment with $\gamma=0.11$, they observe a bulge in the Fourier spectrum of the interfacial displacement (their Figure 12(a)). It spreads approximately from $5 \omega_{0}$ to $10 \omega_{0}$. This bulge is associated with time scales present in the ISW2s that are not necessarily harmonics. In their experiment with $\gamma=0.17$, a weaker bulge is observed in the Fourier spectrum. The amplitude decrease with frequency is smaller after, than before the beam impact.

In the experiments performed in the secondary generation configuration, the ISW2s spectra are closer to the latter case. In fact, the amplitude decrease for short time scales $\left(\omega>5 \omega_{0}\right)$ is smaller after the beam impact. The present experiments are associated with $\gamma \geq 0.15$, while the bulge is 
observed by Mercier et al. ${ }^{24}$ for $\gamma=0.11$. Hence, it could be present only in a short range of pycnocline regimes around the latter value.

\section{STEREO-CORRELATION}

The interfacial wave dynamics has been locally studied in the primary and secondary generation configuration using ultrasonic probes measurements. Stereo correlation measurements performed in the experiments PG052 and SG019 allow to assess and compare the importance of three-dimensional structures associated with these two processes. These two experiments have similar physical parameters to the ones of PG045 and SG033, respectively, for which ISWs trains have been measured with ultrasonic probes.

\section{A. Mean longitudinal structures}

First, the mean transverse isopycnal displacement $\eta_{m}(x, t)=\langle\eta(x, y, t)\rangle_{y}$ is examined in the two regimes and in the steady regime, with $\eta(x, y, t)$ and \langle\rangle$_{y}$, respectively, standing for the interfacial displacement and transverse mean.

In the primary generation case (exp. PG052, Figure 10(a)), the propagation of a $6 \mathrm{~cm}$ deep ISW1 followed by a $2 \mathrm{~cm}$ deep ISW1 is observed. The first (deeper) trough propagates faster $(0.25 \pm 0.03 \mathrm{~m} / \mathrm{s})$ than the second (shallower) trough $(0.14 \pm 0.05 \mathrm{~m} / \mathrm{s})$, what causes a progressive separation of the troughs. The propagation speed of the first ISW1 is close to the theoretical value of $0.26 \mathrm{~m} / \mathrm{s}$ for the linear propagation of an internal wave in an infinitely thin pycnocline. Apart from the progressive separation between the two ISW1s, the mean structure of the train is stable in the course of the propagation. In fact, the number of ISW1s per train is constant and the geometry of a given trough (depth and width) is conserved.

In the secondary generation case (exp. SG019, Figure 10(b)), the periodic propagation of three troughs on the order of centimetres is observed. They propagate at an average speed of 0.11 $\pm 0.03 \mathrm{~m} / \mathrm{s}$. A slight difference in their propagation speeds causes a progressive separation between
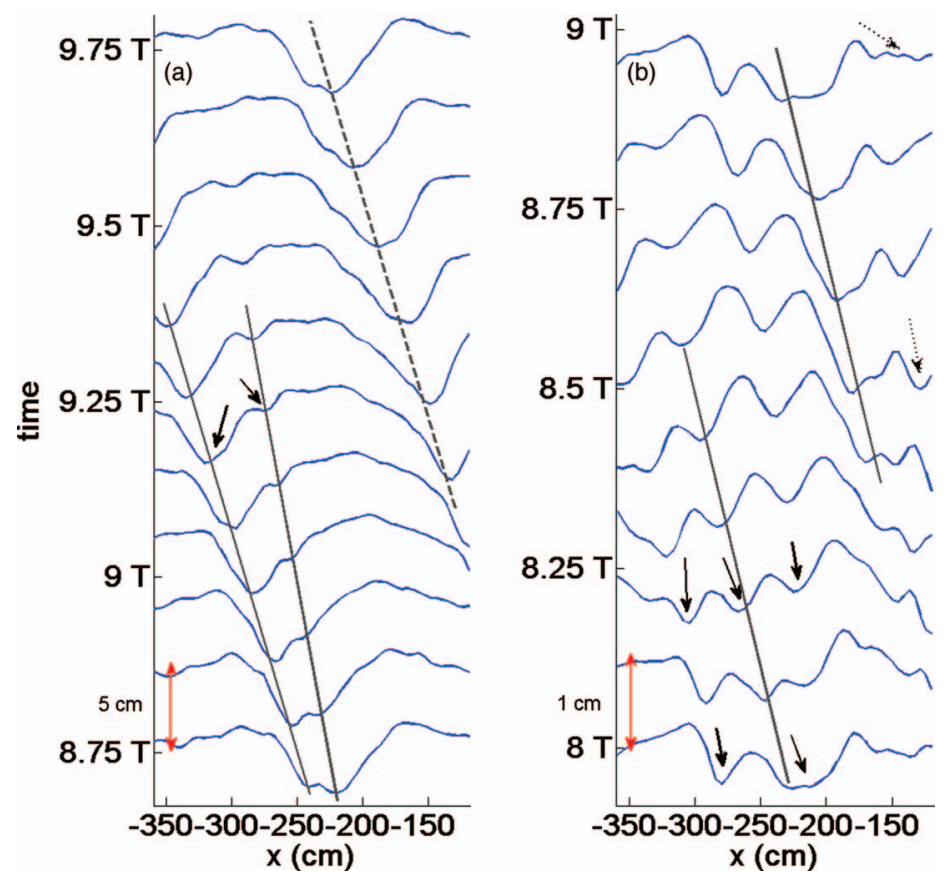

FIG. 10. (a) Transverse mean isopycnal displacement in the pycnocline in PG052 for $t=8.75 ; 8.80 ; \ldots ; 9.75$ T. Solid lines show the propagation of the first and second ISW1s of a train. (b) Transverse mean isopycnal displacement in the pycnocline in SG019 for $t=8 ; 8.125 ; \ldots ; 9$ T. Solid lines show the propagation of ISWs and nonlinear interfacial waves indicated by solid arrows. Dashed arrows indicate a loss of amplitude in a propagating trough. 
the troughs as they propagate. At the right of the field, a progressively decaying propagating trough is observed from $t=8.5 \mathrm{~T}$ to $t=9 \mathrm{~T}$. Overall, the mean interfacial wave structure does not consist in shape-conserving propagating troughs trains that can directly be identified with ISW2s. In fact, the generation of ISW2s may not be as efficient as in the experiment SG033. The value of $\gamma$ that partly controls the efficiency of the secondary generation process is slightly higher in the present experiment $(\gamma=0.21$ versus $\gamma=0.15)$, what can explain a different interfacial dynamics in the two experiments. The interaction of internal wave beams with the pycnocline may generate large amplitude interfacial waves that do not organize into ISW2s trains. Energy transfers from interfacial waves to internal waves propagating in the lower, stratified layer are another explanation for these variations in the longitudinal structure of the troughs. In the case of a stronger pycnocline corresponding to a primary generation configuration, the ISW1s trains are less subject to such energy transfers and therefore conserve their shape.

The term trough, which is more general than ISW2, is used in the remainder of the paper to refer to the interfacial waves observed in exp. SG019. Stereo-correlation measurements with a larger field of view in the longitudinal direction could permit to identify their nature more thoroughly.

The study of the 3D structure of the propagating interfacial waves can provide better insights about their dynamics.

\section{B. 3D structure}

The time evolution of the interfacial displacement field $\eta(x, y, t)$ is studied for the two generation processes.

In the primary generation case, the propagation of one large trough at a speed $0.25 \pm 0.03 \mathrm{~m} / \mathrm{s}$ is observed in Figure 11. Apart from millimetric variations, it has a constant structure in the transverse direction. A second shallower trough with a varying transverse structure is observed. In fact, it is only present in the middle part of the field $(-225 \mathrm{~cm}<y<-75 \mathrm{~cm})$ and vanishes close to the edges of the tank. These two depth-ranked troughs correspond to the ISW1s train characterized using the mean interfacial displacement. In the $1.5 \mathrm{~m}$ plateau separating two ISW1s trains, the interfacial displacement is overall constant in the longitudinal and transverse directions, except from millimetric variations.

The pycnocline displacement in the course of SG019 is shown in Figure 12. Troughs on the order of centimetres and propagating leftwards are observed (blue circles at $t=8.75 \mathrm{~T}$ ). They are in the middle of the field at $t=8 \mathrm{~T}$ and $t=9 \mathrm{~T}$, and correspond to the troughs observed in Figure 10(b). However, transverse variations of comparable magnitude are also present, what makes them harder to identify at all times. These transverse variations are observable in both the troughs and in the plateau separating them. They extend over typically $50 \mathrm{~cm}$ in the transverse direction. A space-time diagram at a given longitudinal position $x=-150 \mathrm{~cm}$ permits to observe the progressive formation of transverse structures (Figure 13). At $t=3 \mathrm{~T}$, a single trough showing millimetric variations in the transverse direction is observed. The magnitude of transverse variations in the interfacial displacement increases with time. It results in a progressive separation of the trough into several troughs of limited transverse extensions. Five troughs reaching the measurement position $x$ $=-150 \mathrm{~cm}$ at different times are observed between $t=8.5 \mathrm{~T}$ and $t=9 \mathrm{~T}$.

Such dynamics is not observed in the primary generation configuration: ISW1s conserve their shape and do not break up in smaller troughs.

\section{Magnitude and sources of the transverse effects}

In order to provide a quantitative evaluation of the transverse effects in the two regimes, the normalized mean transverse deviation $E_{d}=\left\langle\left|\eta(x, y, t)-\eta_{m}(x, t)\right|\right\rangle_{y} / d$ is calculated, where $d$ is the depth of the first trough of the train. One finds $E_{d}=0.08$ for exp. PG052, and $E_{d}=0.14$ for exp. SG019. The magnitude of transverse effects are twice larger in the secondary generation configuration than in the primary generation configuration.

Considering the role of the lower layer can help at understanding better why transverse structures are more substantial in the secondary generation case. 

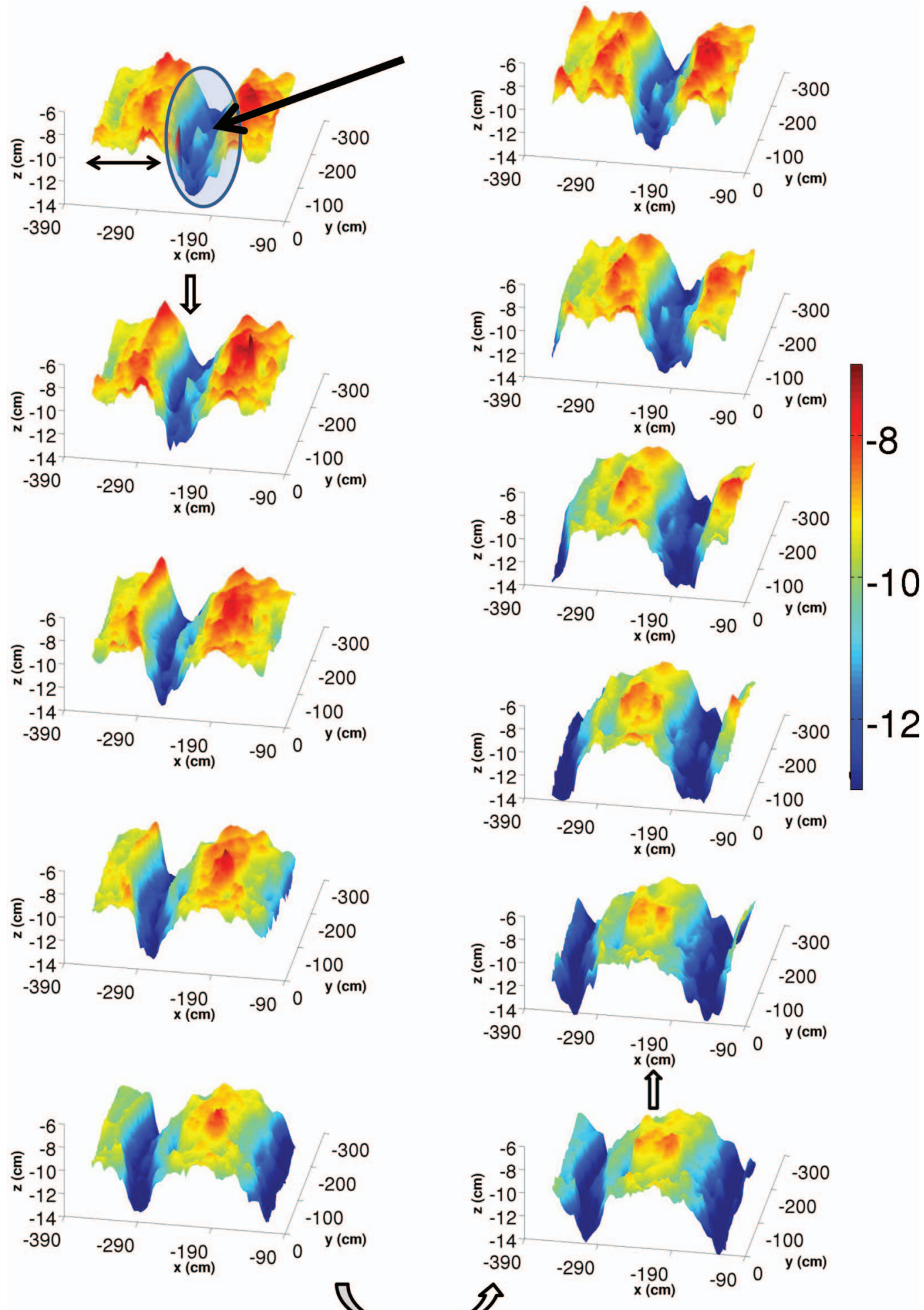

FIG. 11. Stereo correlation measurements of the interfacial displacement in exp. PG052, at $t=8.75 \mathrm{~T}, 8.85 \mathrm{~T}, \ldots, 9.75 \mathrm{~T}$. Time evolves in the counterclockwise direction. The ridge rest position is at $90 \mathrm{~cm}$ to the right of the field. The first ISW1 (blue circle in the first picture) propagates from right to left with a speed of $0.25 \pm 0.03 \mathrm{~m} / \mathrm{s}$. The solid black arrow shows a second ISW1. 

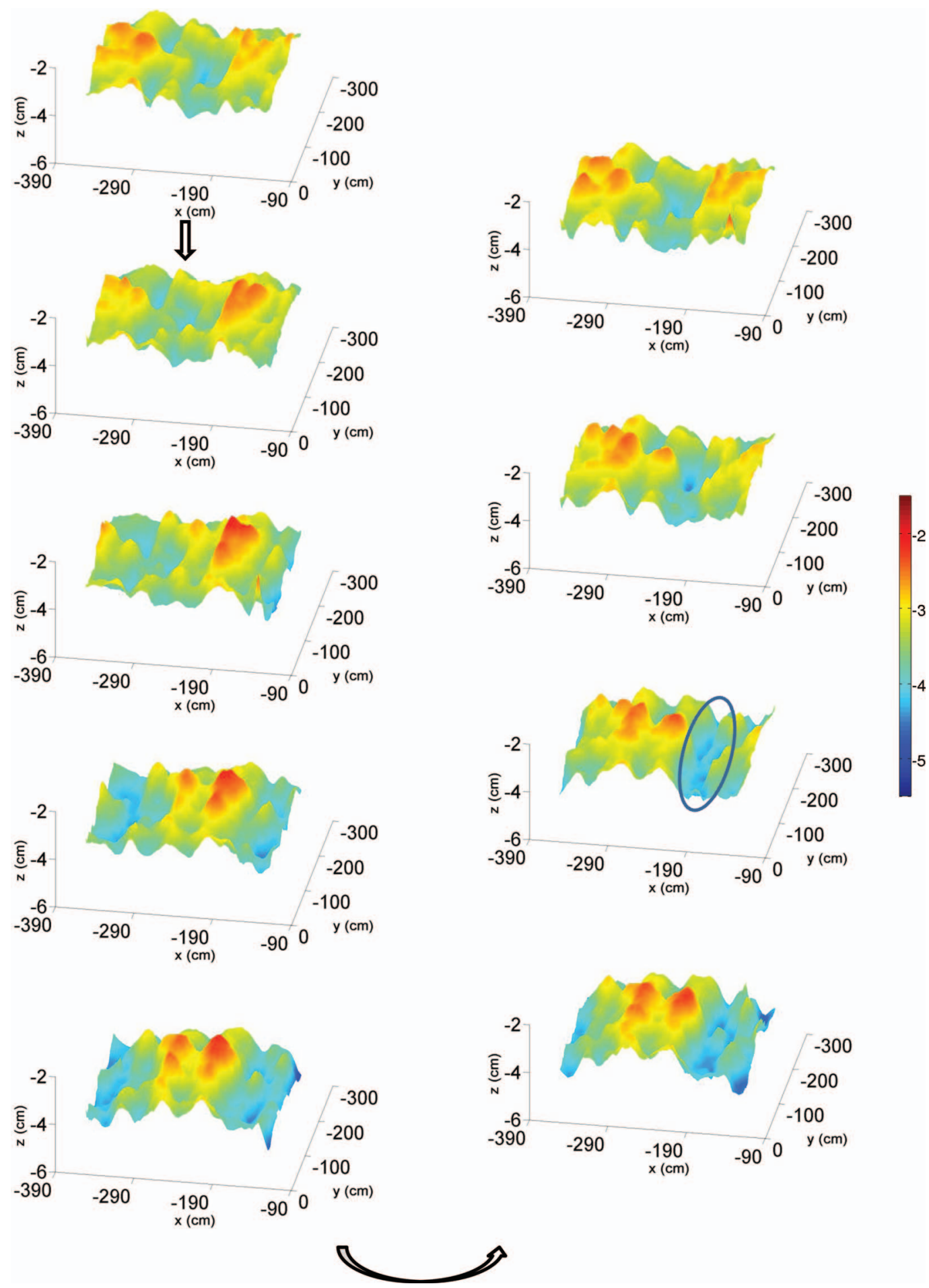

FIG. 12. Stereo correlation measurements of the interfacial displacement in exp. SG019 at $t=8 \mathrm{~T}, 8.125 \mathrm{~T}, \ldots, 9 \mathrm{~T}$. Time evolves in the counterclockwise direction. Troughs propagate to the left of the field at a speed of $0.11 \mathrm{~m} / \mathrm{s}$. The blue circle highlights propagating troughs at $t=8.75 \mathrm{~T}$.

In the case of a moderate pycnocline $(\gamma \approx 0.1)$, interfacial troughs can be generated by the impact of internal wave beams on the pycnocline. In a linear regime $(\epsilon \ll 1)$, the oscillation of the ridge would generate internal wave beams propagating in the direction of the forcing, and non-varying in the transverse direction. In the present configuration, turbulent effects generated by the ridge oscillation 


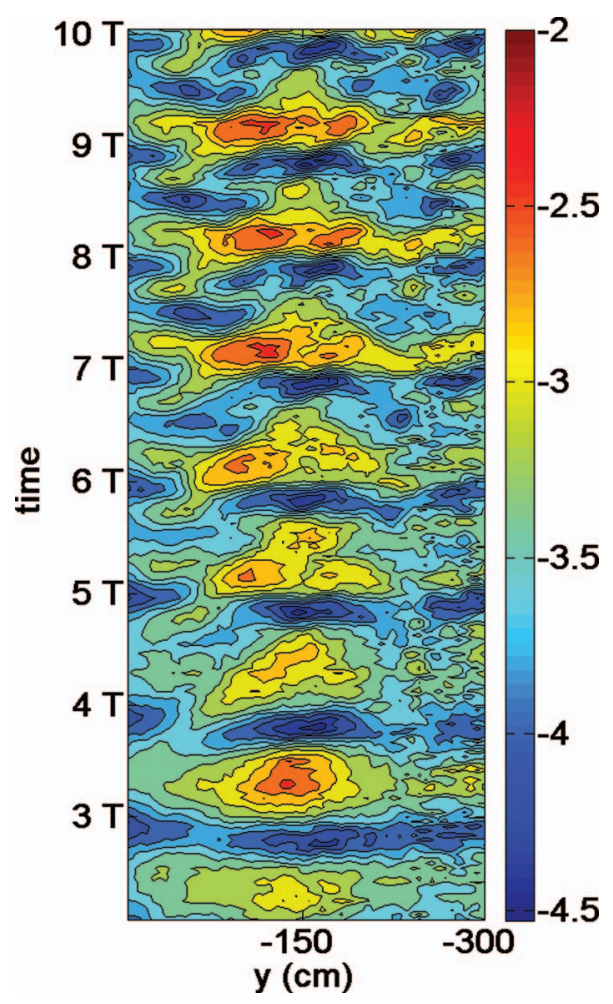

FIG. 13. Space-time diagram of the interfacial displacement at $x=-150 \mathrm{~cm}$ in SG019. Dark blue contours correspond to propagating troughs. Transverse variations in the interfacial wave structure increase with time.

may modify the transverse structure of the topographic internal wave beam. Consequently, transverse variations in the troughs structure are potentially induced by the inhomogeneity of the forcing beam.

As the lower layer is stratified, it can support the propagation of internal waves in various forms after the beam impact. First, an energy fraction of the incoming internal wave beam is reflected down in the form of a scattered beam after the impact (Dossmann, Auclair, and Paci, ${ }^{20}$ Mathur and Peacock, ${ }^{22}$ Grisouard and Staquet ${ }^{31}$ ). Second, as interfacial troughs progress, they may transfer energy to internal waves propagating in the lower layer through a downward leaking process (Gerkema, ${ }^{14}$ Akylas et al. ${ }^{18}$ ).

Nonlinear interactions between the pycnocline and the complex internal wave field in the lower layer can explain the enhancement of transverse effects in the interfacial wave structure as time advances.

Such interactions are limited in the case of a stronger pycnocline corresponding to a primary generation configuration. The oscillation of the ridge generate interfacial waves in the pycnocline, that organize into ISW1s trains due to the nonlinearity/dispersion balance. As the lower layer stratification is too small to support the propagation of internal waves, ISW1s are not likely to undergo a downward leaking of energy and propagate in a stable manner $\left(\mathrm{Gerkema}^{14}\right)$. As the topographic forcing does not vary in the transverse direction, the weak transverse structures observed are possibly induced by turbulent motions and the effects of viscosity close to the tank walls.

\section{CONCLUSION}

Laboratory experiments have been used to study two configurations of internal solitary waves generation inspired by oceanic observations.

First, the primary generation configuration where the fluid consists in two layers of constant densities separated by a sharp pycnocline has been studied. Measurements issued from one probe were used to observe the generation of large amplitude, stable trains of propagating troughs in 
various regimes of nonlinearity and dispersion. Comparisons with previous analytical and numerical studies permitted to associate them with ISW1s.

In the secondary generation configuration where the pycnocline of moderate strength lies on the top of a stratified layer, the interfacial dynamics has been studied using probes disposed longitudinally in one experiment. The generation of a train of troughs by the impact of the internal wave beam on the pycnocline has been observed. The propagating troughs exhibit the dynamics features of ISW2s, including an amplitude decay due to a downward leaking of energy (Gerkema, ${ }^{14}$ Akylas et al. ${ }^{18}$ Dossmann, Auclair, and Paci, ${ }^{20}$ Grisouard and Staquet ${ }^{31}$ ).

Recent developments of the stereo correlation technique have been used to investigate the three-dimensional dynamics of interfacial waves in the two configurations.

ISW1s trains are observed to propagate in a stable manner and are associated with weak transverse structures. On the contrary, the dynamics of the interfacial waves in the secondary generation regime is more complex. In fact, propagating troughs are observed, yet they are not clearly organized in trains of depth-ordered ISW2s. Transverse structures of magnitude on the order of centimetres are observed while they propagate. They are twice as large as in the primary generation case. Interactions of the interfacial troughs with internal waves propagating in the lower layer can be a cause of the generation of transverse structures. Future studies could combine stereo-correlation measurements of the pycnocline displacement with vertical PIV measurements in various $(\mathrm{x}, \mathrm{z})$ planes to assess the role played by internal waves propagating in the lower layer.

It appears that two-dimensional numerical simulations in a vertical plane are relevant to capture the dynamics of ISW1s and of ISW2s to a lesser extent. Three-dimensional simulations are necessary to study in details the interactions between ISW2s and internal waves in the lower layer. In fact, the latter process appears intrinsically linked to the generation of transverse structures in the pycnocline.

\section{ACKNOWLEDGMENTS}

This work has been supported by LEFE-IDAO Programme "ondes et marées internes dans l'océan" (LEFE-IDAO-07/2) and ANR "PIWO" contract (ANR-08-BLAN-0113). Y. Dossmann's Ph.D. thesis was funded by a MNERT scholarship. We thank B. Beaudoin, A. Belleudy, J.-C. Canonici, F. Murguet, H. Schaffner, and V. Valette of the CNRM-GAME (UMR 3589, METEOFRANCE, and CNRS) fluid mechanics laboratory for their kind support. S. Cazin and L. Lacaze from IMFT and S. Viboud from LEGI are also thanked for their help during the experimental campaign, regarding, respectively, the stereo-correlation technique and the ultrasonic probes.

${ }^{1}$ V. C. Tsai, H. Kanamori, and J. Artru, “The morning glory wave of southern California,” J. Geophys. Res. 109, B02307, doi:10.1029/2003JB002596 (2004).

${ }^{2}$ J. W. Rottman and R. Grimshaw, Internal Solitary Waves (Kluwer, 2001), pp. 61-88.

${ }^{3}$ D. Halpern, "Semidiurnal internal tides in Massachusetts bay," J. Geophys. Res. 76, 6573-6584, doi:10.1029/JC076i027p06573 (1971).

${ }^{4}$ A. L. New and R. D. Pingree, "Large-amplitude internal soliton packets in the central Bay of Biscay," Deep Sea Res. A. Oceanogr. Res. Papers 37, 513-524 (1990).

${ }^{5}$ K. H. Helfrich and W. K. Melville, "Long nonlinear internal waves," Annu. Rev. Fluid Mech. 38, 395-425 (2006).

${ }^{6}$ A. L. New and J. C. B. da Silva, "Remote-sensing evidence for the local generation of internal soliton packets in the central Bay of Biscay," Deep Sea Res. 49, 915-934 (2002).

${ }^{7}$ Z. Lai, C. Chen, R. C. Beardsley, B. Rothschild, and R. Tian, "Impact of high-frequency nonlinear internal waves on plankton dynamics in Massachusetts bay," J. Marine Res. 68, 259-281 (2010).

${ }^{8}$ L. S. Kantha and C. A. Clayson, "An improved mixed layer model for geophysical applications," J. Geophys. Res. 99, 25235-25266, doi:10.1029/94JC02257 (1994).

${ }^{9}$ R. D. Pingree and G. T. Mardell, "Solitary internal waves in the Celtic sea," Prog. Oceanogr. 14, 431-441 (1985).

${ }^{10}$ J. Apel, J. Holbrook, A. Liu, and J. Tsai, "The Sulu sea internal soliton experiment," J. Phys. Oceanogr. 15, 1625-1651 (1985).

${ }^{11}$ A. L. New and R. D. Pingree, "Local generation of internal soliton packets in the central Bay of Biscay,” Deep Sea Res. A. Oceanogr. Res. Papers 39, 1521-1534 (1992).

${ }^{12}$ J. C. B. da Silva, A. L. New, and J. M. Magalhaes, "Internal solitary waves in the mozambique channel: Observations and interpretation," J. Geophys. Res. 114, 1-12, doi:10.1029/2008JC005125 (2009).

${ }^{13}$ A. Azevedo, J. C. B. da Silva, and A. L. New, "On the generation and propagation of internal solitary waves in the southern Bay of Biscay,” Deep Sea Res. I: Oceanogr. Res. Papers 53, 927-941 (2006).

${ }^{14}$ T. Gerkema, "Internal and interfacial tides: Beam scattering and local generation of solitary waves," J. Marine Res. 59, 227-255 (2001). 
${ }^{15}$ T. Gerkema, "Development of internal solitary waves in various thermocline regimes: A multi-modal approach," Nonlinear Process. Geophys. 10, 397-405, doi:10.5194/npg-10-397-2003 (2003).

${ }^{16} \mathrm{R}$. Maugé and T. Gerkema, "Generation of weakly nonlinear nonhydrostatic internal tides over large topography: A multi-modal approach,” Nonlinear Process. Geophys. 15, 233-244, doi:10.5194/npg-15-233-2008 (2008).

${ }^{17}$ Y. Dossmann, F. Auclair, and A. Paci, "Topographically induced internal solitary waves in a pycnocline: Primary generation and topographic control," Phys. Fluids 25, 066601 (2013).

${ }^{18}$ T. R. Akylas, R. H. J. Grimshaw, S. R. Clarke, and A. Tabaei, "Reflecting tidal wave beams and local generation of solitary waves in the ocean thermocline," J. Fluid Mech. 593, 297-313 (2007).

${ }^{19}$ N. Grisouard, C. Staquet, T. Gerkema et al., "Generation of internal solitary waves in a pycnocline by an internal wave beam: A numerical study,” J. Fluid Mech. 676, 491 (2011).

${ }^{20}$ Y. Dossmann, F. Auclair, and A. Paci, "Topographically induced internal solitary waves in a pycnocline: Secondary generation and spatial selection criteria," Phys. Fluids 25, 086603 (2013).

${ }^{21}$ D. I. Delisi and I. Orlanski, "On the role of density jumps in the reflexion and breaking of internal gravity waves," J. Fluid Mech. 69, 445-464 (1975).

${ }^{22}$ M. Mathur and T. Peacock, "Internal wave beam propagation in non-uniform stratifications," J. Fluid Mech. 639, 133-152 (2009).

${ }^{23}$ S. Wunsch and A. Brandt, "Laboratory experiments in internal wave interactions with a pycnocline," Exp. Fluids 53, 1663-1679 (2012).

${ }^{24}$ M. J. Mercier, M. Mathur, L. Gostiaux, T. Gerkema, J. M. Magalhaes, J. C. B. da Silva, and T. Dauxois, "Soliton generation by internal tidal beams impinging on a pycnocline: Laboratory experiments," J. Fluid Mech. 704, 37-60 (2012).

${ }^{25} \mathrm{~S}$. J. Ghaemsaidi and T. Peacock, "3D stereoscopic PIV visualization of the axisymmetric conical internal wave field generated by an oscillating sphere," Exp. Fluids 54, 1454 (2013).

${ }^{26}$ J. Hazewinkel, L. Maas, and S. Dalziel, "Tomographic reconstruction of internal wave patterns in a paraboloid," Exp. Fluids 50, 247-258 (2011).

${ }^{27}$ L. Lacaze, A. Paci, E. Cid, S. Cazin, O. Eiff, J. Esler, and E. Johnson, "Wave patterns generated by an axisymmetric obstacle in a two-layer flow," Exp. Fluids 54, 1618 (2013).

${ }^{28}$ T. Maxworthy, “A note on the internal solitary waves produced by tidal flow over a three-dimensional ridge," J. Geophys. Res. 84, 338-346, doi:10.1029/JC084iC01p00338 (1979).

${ }^{29}$ D. Astruc, S. Cazin, E. Cid, O. Eiff, L. Lacaze, P. Robin, F. Toublanc, and I. Caceres, "A stereoscopic method for rapid monitoring of the spatio-temporal evolution of the sand-bed elevation in the swash zone," Coast. Eng. 60, 11-20 (2012).

${ }^{30}$ T. Gerkema, "Nonlinear dispersive internal tides: Generation models for a rotating ocean," Ph.D. thesis, Universiteit Utrecht, 1994.

${ }^{31}$ N. Grisouard and C. Staquet, "Numerical simulations of the local generation of internal solitary waves in the Bay of Biscay,” Nonlinear Process. Geophys. 17, 575-584, doi:10.5194/npg-17-575-2010 (2010).

${ }^{32}$ Y. Dossmann, A. Paci, F. Auclair, and J. W. Floor, "Simultaneous velocity and density measurements for an energy-based approach to internal waves generated over a ridge," Exp. Fluids 51, 1013-1028 (2011).

${ }^{33}$ T. Dauxois and M. Peyrard, Physics of Solitons (Cambridge University Press, New York, 2006). 